\title{
31. PALEOMAGNETIC DATA FROM HOLES 782A, 784A, AND 786A, LEG $125^{1}$
}

\author{
Roger B. Haston, ${ }^{2}$ Laura B. Stokking, ${ }^{3}$ and Jason $\mathrm{Ali}^{4}$
}

\begin{abstract}
During Leg 125 , scientists drilled three holes (782A, 784A, and 786A) in the Izu-Bonin forearc near $31^{\circ} \mathrm{N}$ that had sufficient recovery to obtain paleoinclination data. A total of 169 paleomagnetic samples were analyzed using either alternating field or continuous thermal demagnetization. Unfortunately, poor recovery, complex magnetization in the older sediments, and dipping beds prevented us from obtaining results that were older than middle Miocene. However, six reliable data points (one Pleistocene, three Pliocene, and two Miocene) were obtained. These data agree with past work from around the Philippine Sea plate, which suggest about $20^{\circ}$ of northward translation since the Eocene. This paleomagnetic data set can be used to help constrain models for the origin and history of the Philippine Sea plate.
\end{abstract}

\section{INTRODUCTION}

The Philippine Sea plate is one of the least understood plates on Earth despite several decades of shipborne geologic and geophysical studies. The study of the tectonic features of this plate have heavily influenced our thinking about backarc basin formation, development of forearcs, and subduction processes. Determining the past motion of the Philippine Sea plate is important because the plate forms a major portion of the eastern boundary of Southeast Asia and has played an important role in the development of complex areas such as the Philippine Archipelago. The motion of the plate cannot be determined by traditional plate circuit reconstructions because the plate is surrounded by subduction zones (Fig. 1A). Ranken etal. (1984) concluded that, relative to Eurasia, the Philippine Sea plate is rotating clockwise at a rate of $1.5^{\circ}$ to $2.0 \%$ m.y. about a pole just north of the northern boundary of the plate. How far this motion can be extrapolated into the past is unknown. Paleomagnetism offers us a unique opportunity to determine the past motion of the Philippine Sea plate.

Numerous paleomagnetic studies have been done using Deep Sea Drilling Project (DSDP) cores collected from the Philippine Sea plate (Louden, 1977; Kinoshita, 1980; Keating, 1981; Keating and Herrero, 1981; Bleil, 1982; Niitsuma, 1986), as well as from rocks on the Izu peninsula that are presumed to have been part of the Philippine Sea plate (Hirooka et al., 1985). Figure 1A shows the locations for these DSDP drill holes in the Philippine Sea plate. In general, these data show a decrease in paleolatitude with time, and suggest that the Philippine Sea plate has moved northward $\approx 20^{\circ}$ since the Eocene. Unfortunately, many of the sites have low latitudes; this produces an equatorial ambiguity and makes interpretation of magnetic polarity difficult.

During Leg 125, we drilled several holes in the Izu-Bonin forearc near $31^{\circ} \mathrm{N}$ (Fig. 1B). Recovery was sufficient in three of the holes (782A, 784A, and 786A) to study paleomagnetism. The goal of this paleomagnetic study is to determine paleolatitude for these holes and to compare these new data with previously published results from the Philippine Sea plate. The northerly location of the sites affords us the opportunity to resolve any equatorial ambiguity in the paleolatitudinal record from the Philippine Sea plate. Once the translational history

'Fryer, P., Pearce, J. A., Stokking, L. B., et al., 1992. Proc. ODP, Sci. Results, 125: College Station, TX (Ocean Drilling Program).

${ }^{2}$ Department of Geological Sciences, University of California, Santa Barbara, CA 93106, U.S.A. (Present address: Amoco Production Company, P.O. Box 3092, Houston, TX 77253, U.S.A.)

${ }^{3}$ Ocean Drilling Program, Texas A\&M University, 1000 Discovery Drive, College Station, TX 77845, U.S.A.

${ }^{4}$ Department of Oceanography, University of Southampton, S09 5NH Southampton, United Kingdom. for the plate is established, this information can be used to help constrain models of the evolution of the Philippine Sea plate.

\section{PALEOMAGNETIC METHOD}

One hundred and sixty-nine paleomagnetic samples were analyzed from Holes 782A, 784A, and 786A using either $7-\mathrm{cm}^{3}$ plastic boxes or $10-\mathrm{cm}^{3}$ pyrex tubes. Samples were analyzed in the University of California (Santa Barbara) paleomagnetic laboratory within a magnetically shielded room. Each sample was individually demagnetized using either stepwise alternating field (AF) or continuous thermal demagnetization (Dunn and Fuller, 1984) and measured on a Molyneux Molspin ${ }^{\star}$ spinner magnetometer or a $2 \mathrm{G}$ Enterprises $^{\text {(t) }}$ three-axis cryogenic SQUID magnetometer. Stable directions were selected using equal area and Zijderveld (1967) vector end-point plots. All of the cores are azimuthally unoriented; thus, the standard statistics developed by Fisher (1953) cannot be used. Instead, the inclination-only statistics of McFadden and Reid (1982) were used to calculate mean inclinations and the associated error limits. Because of the poor quality of the data and the extensive number of cores that were affected by drilling disturbance, relatively coarse time intervals were used to calculate of mean inclination points. Thus, the error limits for the age of each averaged point is substantial.

\section{PALEOMAGNETIC DATA}

\section{Hole 782A}

Seventy-one samples that ranged in age from Pleistocene to middle Miocene were measured from cores between 39.58 and 381.86 mbsf. Below this level, recovery was too sparse to yield reliable paleomagnetic results. Both continuous thermal and stepwise AF demagnetizations were used to demagnetize the samples. Many of the samples yield easily defined single components of magnetization (Fig. 2A), whereas others show no characteristic remanent magnetization direction (Fig. 2B). Table 1 lists the paleomagnetic data from Hole $782 \mathrm{~A}$. Of the 71 samples collected, 53 yield reliable characteristic directions. Figure 3 shows inclination data from the samples of Hole $782 \mathrm{~A}$ vs. depth. Also shown in the figure is an estimate of drilling disturbance in the cores, averaged over 5-m intervals.

The interval between 39 and 56 mbsf yield consistent magnetic inclination values in both normal and reversely magnetized samples. However, between 56 and 140 mbsf, the characteristic magnetic directions from the samples show a large amount of scatter. The amount of drilling disturbance in the interval between 39 and $56 \mathrm{mbsf}$ can be characterized as slight, with sporadic zones of moderate disturbance. However, between 56 and 140 mbsf, the amount of 


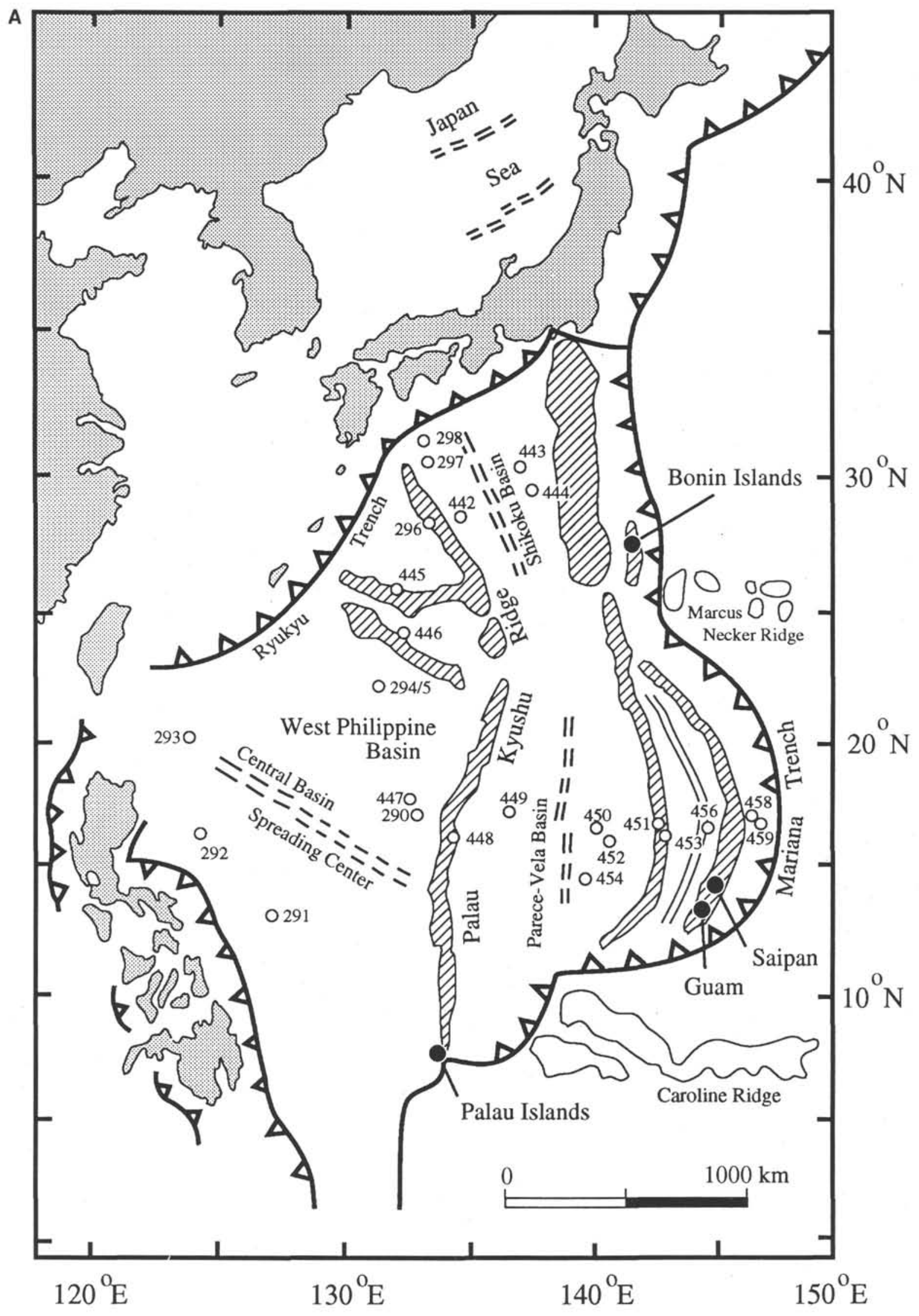

Figure 1. A. Major features of the Philippine Sea plate showing the locations of the DSDP drill sites (open circles). Bold lines with teeth indicate plate boundaries. B. Location of the Leg 125 drill holes used in this study. Contour intervals in kilometers. 
B

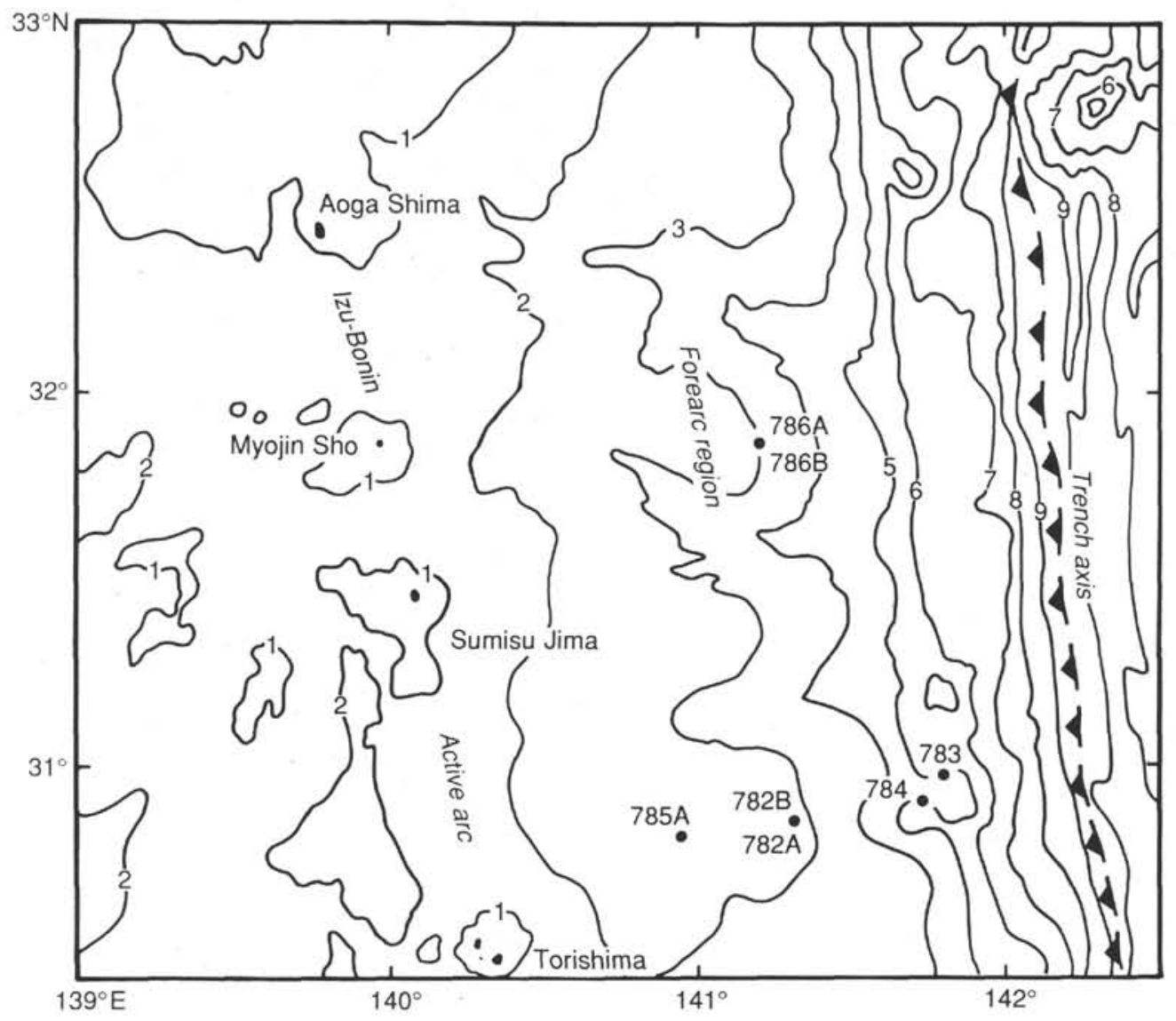

Figure 1 (continued).

disturbance is much more significant. Although care was taken to try to select samples away from disturbed zones, the lack of internal structure within many of the cores made determination of disturbance difficult. It seems likely that the widely scattered results from this interval may be due to deformation of the cores during drilling and should be omitted from further analysis. The seven samples in the late Pleistocene interval between 39 and $56 \mathrm{mbsf}$ yield a mean inclination of $I=45.8^{\circ}\left(\alpha_{95}=\right.$ $7.2^{\circ}, \kappa=22.0$ ), which is not significantly different from the inclination predicted by the dipole field at the site latitude.

Between 140 and $280 \mathrm{mbsf}$, the amount of drilling disturbance decreases and can be characterized as being slight to moderate. In this same interval, the scatter in the measured inclination values also decreases. Both normal and reversely magnetized samples were measured and show a similar amount of scatter. Although several of the normal samples are near the predicted present inclination, there is no clear evidence to suggest that any of the samples have been remagnetized. Twenty samples from the upper Miocene sediments (between 142 and 250 mbsf) yield a mean inclination of $I=41.7^{\circ}\left(\alpha_{95}=6.0^{\circ}, \kappa=32.0\right)$, which is slightly shallower than the present dipole field.

Eleven samples from middle Miocene sediments (between 250 and $280 \mathrm{mbsf}$ ), four normal and seven reversed, yield stable directions. The mean of these 11 samples is $I=40.8^{\circ}\left(\alpha_{95}=7.0^{\circ}, \kappa=52\right)$, which is also shallower than the predicted present field inclination value. Below $280 \mathrm{mbsf}$, no reliable data exist because of the poor core recovery and high drilling disturbance.

\section{Hole 784A}

A total of 22 samples were measured from cores taken between 13.09 and $311.58 \mathrm{mbsf}$; these ranged in age from Pleistocene to
Miocene. Continuous thermal demagnetization was used to demagnetize all of the samples from this hole. Of the 22 samples collected, only 19 yield characteristic remanent magnetic directions. In general, the samples were well behaved and carried a single magnetic component (Fig. 4A). Table 2 lists all of the paleomagnetic data from Hole 784 A. Poor recovery, drilling disturbance, tilted beds $\left(35^{\circ}\right.$ at 190 mbsf), and complex magnetization behavior (Fig. 4B) made interpretation of any paleomagnetic data below $85 \mathrm{mbsf}$ impossible. Figure 5 shows the inclination data, along with drilling disturbance vs. depth, for Hole 784A. The upper $30 \mathrm{~m}$ of the hole shows a high amount of drilling disturbance; inclination data from this interval are scattered. It seems likely that the magnetization in these samples was affected by drilling disturbance, and that these directions are not accurate recordings of Earth's field during the Pleistocene. Seven samples (Pliocene in age) between 40 and $85 \mathrm{mbsf}$ yield characteristic remanent magnetizations that have both normal and reversed polarity. The drilling disturbance in this interval is negligible to slight. The mean inclination for these seven samples is $I=38.5^{\circ}\left(\alpha_{95}=14.0^{\circ}, \kappa=29.0\right)$. This is slightly shallower than the predicted present dipole field inclination value, but is indistinguishable at the $95 \%$ level of confidence.

\section{Hole 786A}

Seventy-six samples were measured from cores between 0 and 102 mbsf, and ranged in age from early Pleistocene to late Eocene. Basaltic flows and breccias were recovered below this point; these were unsuitable for this study. Both continuous thermal and stepwise AF demagnetizations were used to demagnetize the samples. Table 3 lists all of the paleomagnetic data for Hole 786A. Many of the samples 

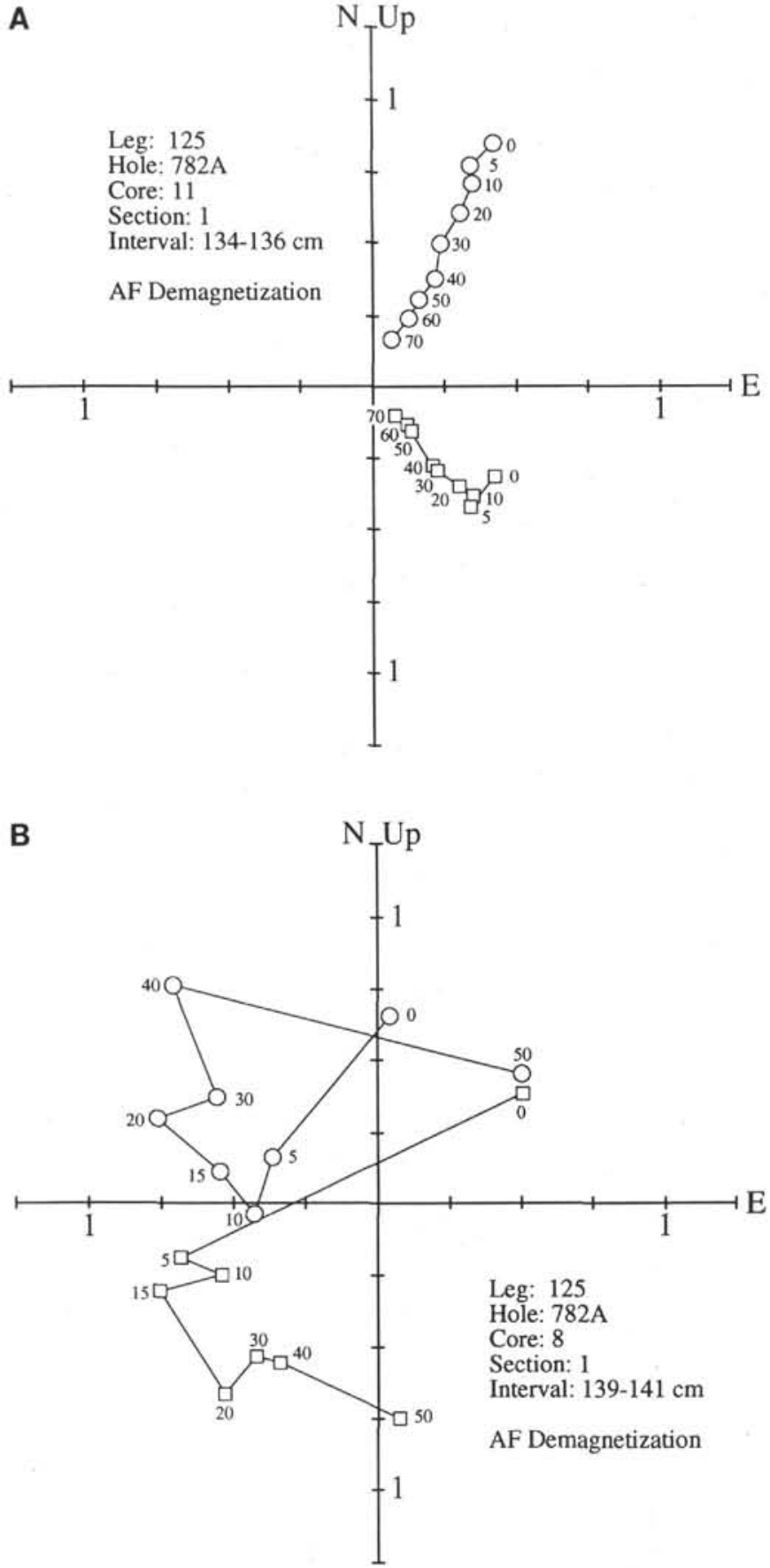

Figure 2. Typical orthogonal vector plots for samples from Hole 782A. A. Single component behavior. B. No characteristic directional behavior.

contain easily identified single components of magnetization (Fig. 6A), although the magnetization behavior of the older samples is much more complex (Fig. 6B).

Hole 786A held the greatest promise of obtaining a good inclination vs. time record of any of the holes drilled during Leg 125, mainly because sediments were recovered that dated back to the Eocene. However, poor recovery, drilling disturbance, and moderately to steeply dipping beds made acquisition of data older than early Pliocene impossible. Figure 7 shows the inclination data and drilling disturbance vs. depth for Hole 786A.

A total of 13 samples (nine reversed and four normal polarity) yield characteristic remanent magnetic directions from sediments that are late Pliocene in age. One sample $(125-786 \mathrm{~A}-2 \mathrm{H}-1,44 \mathrm{~cm})$ was rejected because it has an anomalously low inclination $\left(-12^{\circ}\right)$; clearly, the site was not at the equator during the late Pliocene. Although there is a relatively large spread in the data, the majority cluster around the predicted present dipole field (Fig. 7), which has a mean inclination of $I=51.3^{\circ}\left(\alpha_{95}=9.0^{\circ}, \kappa=23.0\right)$. Individual samples yield easily defined single components of magnetization (Fig. 7); yet, the overall results are dispersed. We are not clear about what caused the scatter in these data, although the cores are slightly to moderately disturbed.

Fifteen samples from rocks that are early Pliocene in age yield single components of magnetization that have both normal (6) and reversed (10) polarities after both $\mathrm{AF}$ and continuous thermal demagnetization. The samples are well grouped and have a mean inclination of $I=40.0^{\circ}\left(\alpha_{95}=6.0^{\circ}, \kappa=36.0\right)$, which is slightly lower than the predicted dipole inclination for the site.

Forty-six samples from rocks older than early Pliocene were demagnetized using both $\mathrm{AF}$ and continuous thermal demagnetization. Individually, 25 of the samples yield well-defined, characteristic, remanent magnetic directions; however, as a group, the samples are scattered and range in inclination between $0^{\circ}$ and $50^{\circ}$ (Fig. 7). Those sediments older than early Pliocene have tilted significantly, reaching up to $40^{\circ}-50^{\circ}$ in the sediments of late Eocene age. Because the cores are azimuthally unoriented and accurate strike is unknown in the area, there is no way to correct the inclination data back to their pre-tilting position. Furthermore, below $65 \mathrm{mbsf}$, the drilling disturbance is moderate to high. For these reasons, we rejected all of the data below 29 mbsf as unreliable.

\section{DISCUSSION}

Unfortunately, our original goal of establishing a latitude vs. time record back to the Eocene for this portion of the Izu-Bonin arc have not been achieved because of complex magnetic behavior, poor recovery, and significant drilling disturbance in many of the cores. However, six new time points (Pliocene from Hole 784A, late and middle Miocene from Hole 782A, and late and early Pliocene from Hole 786A) can be added to the paleomagnetic database from the Philippine Seaplate. These new data are summarized in Table 4. Many deep sea cores have been shown to have inclination flattening due to compaction (Celaya and Clement, 1988; Arason and Levi, 1990; and others). Whether the shallowing of the inclination is purely tectonic or has a component of flattening due to compaction is unknown. Arason and Levi (1990) showed that inclination flattening was likely to occur in areas where the sedimentation rate was quite high; the sedimentation rate in the younger sediments drilled during Leg 125 was fairly low.

Figure 8 shows the paleolatitude data compiled from other studies from around the Philippine Sea plate, along with the data from this study. These data are plotted as change in latitude vs. time. Change in latitude is defined as the observed paleolatitude minus the site latitude. This has the beneficial effect of normalizing all of the sites to a common latitude and allows for observations of the overall motion of the plate to be observed. Although different locations on the Philippine Sea plate may have had different translational histories if the location of the finite pole of rotation of the Philippine Sea plate relative to the geomagnetic pole was near the plate, the resolution of the data are not sufficient for detecting these differences. Also plotted are the on-land paleomagnetic studies (taken from Haston and Fuller, 1990). The data suggest that the plate has moved as much as $20^{\circ}$ northward since the Eocene.

\section{ACKNOWLEDGMENTS}

We thank Mike Fuller, Bob Dunn, Stan Cisowski, and Doug Smith for their help with the analysis of the samples and the exploration of ideas. 
Table 1. Paleomagnetic data from Hole 782A, Leg 125.

\begin{tabular}{|c|c|c|c|c|c|c|}
\hline Sample (cm) & $\begin{array}{l}\text { Depth } \\
\text { (mbsf) }\end{array}$ & $\begin{array}{c}\text { Demagnetization } \\
\text { type }^{\mathrm{a}}\end{array}$ & $\begin{array}{c}\text { Maximum } \\
\text { demagnetization }\end{array}$ & $\begin{array}{l}\text { Inclination } \\
\text { (degrees) }\end{array}$ & $\begin{array}{c}\text { Declination } \\
\text { (degrees) }\end{array}$ & Comments \\
\hline \multicolumn{7}{|l|}{$125-782 \mathrm{~A}-$} \\
\hline $5 \mathrm{H}-1,128$ & 39.58 & $\mathrm{AF}$ & 50 & -46.0 & 202.0 & \\
\hline $5 \mathrm{H}-2,138$ & 41.18 & $\mathrm{AF}$ & 50 & -51.0 & 197.0 & \\
\hline $5 \mathrm{H}-3,115$ & 42.45 & CTD & $565^{\circ}$ & 42.0 & 294.0 & \\
\hline $5 \mathrm{H}-3,138$ & 42.68 & $\mathrm{AF}$ & 40 & & & Unstable \\
\hline $5 \mathrm{H}-4,138$ & 44.18 & $\mathrm{AF}$ & 50 & 36.0 & 290.0 & \\
\hline $6 \mathrm{H}-4,103$ & 53.33 & $\mathrm{AF}$ & 50 & 48.0 & 311.0 & \\
\hline $6 \mathrm{H}-5,101$ & 54.81 & $\mathrm{AF}$ & 50 & -52.0 & 152.0 & \\
\hline $6 \mathrm{H}-6,101$ & 56.31 & $\mathrm{AF}$ & 50 & -46.0 & 128.0 & \\
\hline $6 \mathrm{H}-7,67$ & 57.47 & $\mathrm{AF}$ & 30 & & & Unstable \\
\hline $7 \mathrm{H}-1,79$ & 58.09 & $\mathrm{AF}$ & 50 & 16.0 & 205.0 & \\
\hline $7 \mathrm{H}-7,65$ & 66.95 & $\mathrm{AF}$ & 50 & -68.0 & 62.0 & \\
\hline $8 \mathrm{H}-1,139$ & 68.19 & $\mathrm{AF}$ & 50 & & & \\
\hline $8 \mathrm{H}-2,139$ & 69.69 & $\mathrm{AF}$ & 50 & -17.0 & 86 & \\
\hline $8 \mathrm{H}-3,139$ & 71.19 & $\mathrm{AF}$ & 50 & -30.0 & 326 & \\
\hline $8 \mathrm{H}-4,139$ & 72.69 & $\mathrm{AF}$ & 50 & -34.0 & 340.0 & \\
\hline $8 \mathrm{H}-5,41$ & 73.21 & CTD & 50 & -74.0 & 319.0 & \\
\hline $9 \mathrm{H}-3,130$ & 80.60 & $\mathrm{AF}$ & 50 & 12.0 & 171.0 & \\
\hline $9 \mathrm{H}-4,130$ & 82.10 & $\mathrm{AF}$ & 50 & 71.0 & 229.0 & \\
\hline $9 \mathrm{H}-5,130$ & 83.60 & $\mathrm{AF}$ & 50 & & & Unstable \\
\hline $9 \mathrm{H}-6,130$ & 85.10 & $\mathrm{AF}$ & 40 & & & Unstable \\
\hline $11 \mathrm{H}-1,134$ & 97.04 & $\mathrm{AF}$ & 70 & -53.0 & 139.0 & \\
\hline $11 \mathrm{H}-2,82$ & 98.02 & AF & 50 & -56.0 & 196.0 & \\
\hline $11 \mathrm{H}-3,63$ & 99.33 & AF & 70 & 50.0 & 140.0 & \\
\hline $12 \mathrm{H}-2,122$ & 108.02 & AF & 50 & 4.0 & 40.0 & \\
\hline $12 \mathrm{H}-3,122$ & 109.52 & $\mathrm{AF}$ & 70 & -44.0 & 33.0 & \\
\hline $13 \mathrm{H}-1,140$ & 116.40 & $\mathrm{AF}$ & 50 & & & Unstable \\
\hline $13 \mathrm{H}-3,138$ & 119.38 & $\mathrm{AF}$ & 50 & -45.0 & 11.0 & \\
\hline $14 \mathrm{H}-1,128$ & 125.88 & $\mathrm{AF}$ & 50 & 10.0 & 346.0 & \\
\hline $14 \mathrm{H}-2,128$ & 127.38 & $\mathrm{AF}$ & 50 & 2.0 & 220.0 & \\
\hline $14 \mathrm{H}-3,128$ & 128.88 & $\mathrm{AF}$ & 50 & 11.0 & 227.0 & \\
\hline $14 \mathrm{H}-4,128$ & 130.38 & $\mathrm{AF}$ & 50 & 22.0 & 336.0 & \\
\hline $15 \mathrm{H}-1,130$ & 135.60 & $\mathrm{AF}$ & 50 & 24.0 & 217.0 & \\
\hline $15 \mathrm{H}-2,130$ & 137.10 & $\mathrm{AF}$ & 50 & -8.0 & 55.0 & \\
\hline $15 \mathrm{H}-3,130$ & 138.60 & $\mathrm{AF}$ & 50 & & & Unstable \\
\hline $16 \mathrm{H}-1,108$ & 144.98 & $\mathrm{AF}$ & 50 & 38.0 & 188.0 & \\
\hline $16 \mathrm{H}-2,107$ & 146.47 & $\mathrm{AF}$ & 50 & 54.0 & 56.0 & \\
\hline $16 \mathrm{H}-3,106$ & 147.96 & $\mathrm{AF}$ & 50 & 054.0 & 109.0 & \\
\hline $17 \mathrm{H}-1,130$ & 154.90 & $\mathrm{AF}$ & 50 & 30.0 & 324.0 & \\
\hline $17 \mathrm{H}-2,130$ & 156.40 & $\mathrm{AF}$ & 50 & -37.0 & 34.0 & \\
\hline $19 \mathrm{H}-1,120$ & 174.10 & $\mathrm{AF}$ & 50 & 41.0 & 15.0 & \\
\hline $19 \mathrm{H}-2,108$ & 175.48 & $\mathrm{AF}$ & 50 & 25.0 & 110.0 & \\
\hline $19 \mathrm{H}-3,120$ & 177.10 & $\mathrm{AF}$ & 50 & 52.0 & 279.0 & \\
\hline $22 \mathrm{H}-1,108$ & 202.88 & $\mathrm{AF}$ & 70 & -46.0 & 128.0 & \\
\hline $23 \mathrm{H}-2,105$ & 214.05 & $\mathrm{AF}$ & 50 & 38.0 & 214.0 & \\
\hline $23 \mathrm{H}-6,116$ & 220.16 & $\mathrm{AF}$ & 50 & 54.0 & 5.0 & \\
\hline $24 \mathrm{H}-1,49$ & 221.49 & $\mathrm{AF}$ & 50 & 58.0 & 161.0 & \\
\hline $24 \mathrm{H}-2,45$ & 222.95 & $\mathrm{AF}$ & 50 & 44.0 & 285.0 & \\
\hline $25 \mathrm{H}-1,120$ & 231.90 & $\mathrm{AF}$ & 70 & -51.0 & 150.0 & \\
\hline $25 \mathrm{H}-2,120$ & 233.40 & $\mathrm{AF}$ & 60 & -39.0 & 200.0 & \\
\hline $25 \mathrm{H}-3,120$ & 234.90 & $\mathrm{AF}$ & 70 & -44.0 & 14.0 & \\
\hline $25 \mathrm{H}-4,135$ & 236.55 & $\mathrm{AF}$ & 30 & & & Unstable \\
\hline $25 \mathrm{H}-5,136$ & 238.06 & $\mathrm{AF}$ & 70 & 59.0 & 240.0 & \\
\hline $25 \mathrm{H}-6,105$ & 239.25 & $\mathrm{AF}$ & 70 & & & Unstable \\
\hline $26 \mathrm{H}-1,43$ & 240.73 & $\mathrm{AF}$ & 50 & & & Unstable \\
\hline $26 \mathrm{H}-2,29$ & 242.09 & $\mathrm{AF}$ & 50 & 37.0 & 238.0 & \\
\hline $26 \mathrm{H}-3,66$ & 243.96 & $\mathrm{AF}$ & 50 & -42.0 & 44.0 & \\
\hline $26 \mathrm{H}-4,57$ & 245.37 & $\mathrm{AF}$ & 70 & & & Unstable \\
\hline $26 \mathrm{H}-5,68$ & 246.98 & $\mathrm{AF}$ & 70 & 40.0 & 256.0 & \\
\hline $26 \mathrm{H}-5,68$ & 246.98 & $\mathrm{AF}$ & 70 & 40.0 & 256.0 & \\
\hline $26 \mathrm{H}-6,70$ & 248.50 & $\mathrm{AF}$ & 40 & & & Unstable \\
\hline $27 \mathrm{H}-1,145$ & 251.45 & $\mathrm{AF}$ & 70 & & & Unstable \\
\hline $27 \mathrm{H}-2,124$ & 252.74 & $\mathrm{AF}$ & 50 & 22.0 & 86.0 & \\
\hline $27 \mathrm{H}-3,59$ & 253.59 & $\mathrm{AF}$ & 40 & & & Unstable \\
\hline $28 \mathrm{H}-1,113$ & 260.73 & $\mathrm{AF}$ & 60 & -42.0 & 239.0 & \\
\hline $28 \mathrm{H}-2,112$ & 262.22 & $\mathrm{AF}$ & 60 & 46.0 & 198.0 & \\
\hline $28 \mathrm{H}-3,115$ & 263.75 & $\mathrm{AF}$ & 50 & & & Unstable \\
\hline $28 \mathrm{H}-4,76$ & 264.86 & $\mathrm{AF}$ & 50 & -45.0 & 13.0 & \\
\hline $28 \mathrm{H}-5,108$ & 266.68 & $\mathrm{AF}$ & 70 & -45.0 & 18.0 & \\
\hline $28 \mathrm{H}-6,141$ & 268.51 & $\mathrm{AF}$ & 60 & -35.0 & 111.0 & \\
\hline $29 \mathrm{H}-1,88$ & 270.08 & $\mathrm{AF}$ & 60 & -30.0 & 341.0 & \\
\hline $29 \mathrm{H}-2,139$ & 272.09 & $\mathrm{AF}$ & 60 & -45.0 & 158.0 & \\
\hline $29 \mathrm{H}-3,13$ & 272.33 & $\mathrm{AF}$ & 60 & -33.0 & 272.0 & \\
\hline $29 \mathrm{H}-4,78$ & 274.48 & $\mathrm{AF}$ & 70 & 55.0 & 17.0 & \\
\hline $29 \mathrm{H}-5,66$ & 275.86 & $\mathrm{AF}$ & 70 & 38.0 & 239.0 & \\
\hline $29 \mathrm{H}-6,16$ & 276.86 & $\mathrm{AF}$ & 50 & & & Unstable \\
\hline $41 \mathrm{H}-2,16$ & 381.86 & CTD & $520^{\circ}$ & & & Unstable \\
\hline
\end{tabular}

${ }^{\mathrm{a}} \mathrm{AF}=$ alternating field $\mathrm{CTD}=$ continuous thermal demagnetization. 
Table 2. Paleomagnetic data from Hole 784A, Leg 125.

\begin{tabular}{|c|c|c|c|c|c|c|}
\hline Sample (cm) & $\begin{array}{l}\text { Depth } \\
\text { (mbsf) }\end{array}$ & $\begin{array}{c}\text { Demagnetization } \\
\text { type }^{\mathrm{a}}\end{array}$ & $\begin{array}{c}\text { Maximum } \\
\text { demagnetization }\end{array}$ & $\begin{array}{l}\text { Inclination } \\
\text { (degrees) }\end{array}$ & $\begin{array}{l}\text { Declination } \\
\text { (degrees) }\end{array}$ & Comments \\
\hline \multicolumn{7}{|l|}{$125-784 \mathrm{~A}$ - } \\
\hline $3 \mathrm{H}-2,69$ & 13.09 & CTD & $526^{\circ}$ & 31.0 & 239.0 & \\
\hline $4 \mathrm{H}-2,90$ & 22.80 & CTD & $536^{\circ}$ & -25.0 & 323.0 & \\
\hline $4 \mathrm{H}-3,90$ & 24.30 & CTD & $521^{\circ}$ & 13.0 & 322.0 & \\
\hline $6 \mathrm{H}-2,33$ & 41.33 & CTD & $516^{\circ}$ & 44.0 & 39.0 & \\
\hline $6 \mathrm{H}-3,33$ & 42.88 & CTD & $539^{*}$ & & & Unstable \\
\hline $8 \mathrm{H}-3,43$ & 62.23 & CTD & $526^{\circ}$ & -41.0 & 354.0 & \\
\hline $8 \mathrm{H}-4,43$ & 63.37 & CTD & $522^{\circ}$ & -30.0 & 30.0 & \\
\hline $9 \mathrm{H}-3,26$ & 71.76 & CTD & $502^{\circ}$ & 29.0 & 47.0 & \\
\hline $9 \mathrm{H}-4,26$ & 73.26 & CTD & $501^{*}$ & 30.0 & 36.0 & \\
\hline $10 \mathrm{H}-2,92$ & 80.52 & CTD & $501^{*}$ & 46.0 & 150.0 & \\
\hline $10 \mathrm{H}-4,92$ & 83.52 & CTD & $502^{\circ}$ & -55.0 & 188.0 & \\
\hline $12 \mathrm{H}-1,105$ & 98.45 & CTD & $501^{*}$ & 70.0 & 53.0 & \\
\hline $12 \mathrm{H}-2,35$ & 99.25 & CTD & $502^{*}$ & 36.0 & 61.0 & \\
\hline $15 \mathrm{H}-6,110$ & 135.00 & CTD & $502^{*}$ & 14.0 & 56.0 & \\
\hline $16 \mathrm{H}-3,134$ & 140.34 & CTD & $476^{\circ}$ & -15.0 & 127.0 & \\
\hline $16 \mathrm{H}-6,134$ & 144.84 & CTD & $478^{*}$ & -3.0 & 253.0 & \\
\hline $18 \mathrm{H}-1,134$ & 156.13 & CTD & $512^{\circ}$ & 32.0 & 218.0 & \\
\hline $18 \mathrm{H}-6,83$ & 164.08 & CTD & $501^{\circ}$ & 10.0 & 157.0 & \\
\hline $22 \mathrm{H}-3,15$ & 197.15 & CTD & $508^{*}$ & -18.5 & 291.0 & \\
\hline $29 \mathrm{H}-4,94$ & 266.84 & CTD & $501^{*}$ & -45.0 & 165.0 & \\
\hline $29 \mathrm{H}-5,96$ & 268.36 & CTD & $502^{\circ}$ & 35.0 & 298.0 & \\
\hline $29 \mathrm{H}-6,41$ & 269.31 & CTD & $501^{*}$ & 10.0 & 298.0 & \\
\hline $34 \mathrm{H}-1,70$ & 310.40 & CTD & $531^{\circ}$ & & & Unstable \\
\hline $34 \mathrm{H}-2,38$ & 311.58 & CTD & $501^{*}$ & & & Unstable \\
\hline
\end{tabular}

${ }^{a} \mathrm{CTD}=$ continuous thermal demagnetization.

Table 3. Paleomagnetic data from Hole 786A, Leg 125.

\begin{tabular}{|c|c|c|c|c|c|c|}
\hline Sample (cm) & $\begin{array}{l}\text { Depth } \\
\text { (mbsf) }\end{array}$ & $\begin{array}{l}\text { Demagnetization } \\
\text { type }^{a}\end{array}$ & $\begin{array}{c}\text { Maximum } \\
\text { demagnetization }\end{array}$ & $\begin{array}{l}\text { Inclination } \\
\text { (degrees) }\end{array}$ & $\begin{array}{l}\text { Declination } \\
\text { (degrees) }\end{array}$ & Comments \\
\hline \multicolumn{7}{|l|}{$125-786 \mathrm{~A}-$} \\
\hline IH-I. 23 & 0.23 & $\mathrm{AF}$ & 20 & & & Unstable \\
\hline $1 \mathrm{H}-2,9$ & 1.59 & $\mathrm{AF}$ & 50 & -47.0 & 14.0 & \\
\hline $1 \mathrm{H}-2,32$ & 1.82 & CTD & $448^{*}$ & -42.7 & 14.7 & \\
\hline $1 \mathrm{H}-3,6$ & 3.06 & $\mathrm{AF}$ & 50 & -45.0 & 18.0 & \\
\hline $1 \mathrm{H}-3,32$ & 3.32 & CTD & $544^{*}$ & -30.1 & 12.9 & \\
\hline $1 \mathrm{H}-4,7$ & 4.57 & $\mathrm{AF}$ & 50 & -49.0 & 354.0 & \\
\hline IH- 4,32 & 4.82 & CTD & $475^{\circ}$ & 39.6 & 190.5 & \\
\hline $1 \mathrm{H}-5,7$ & 6.07 & AF & 50 & 70.0 & 175.0 & \\
\hline $1 \mathrm{H}-6,7$ & 7.57 & $\mathrm{AF}$ & 50 & 66.0 & 150.0 & \\
\hline $1 \mathrm{H}-7,14$ & 9.14 & $\mathrm{AF}$ & 50 & -66.0 & 7.0 & \\
\hline $2 \mathrm{H}-\mathrm{I}, 42$ & 10.12 & $\mathrm{AF}$ & 100 & -11.9 & 219.0 & \\
\hline $2 \mathrm{H}-2,15$ & 11.35 & $\mathrm{AF}$ & 50 & & & Unstable \\
\hline $2 \mathrm{H}-2,22$ & 11.42 & CTD & $547^{\circ}$ & 53.3 & 23.0 & \\
\hline $2 \mathrm{H}-3,42$ & 13.12 & $\mathrm{AF}$ & 30 & & & Unstable \\
\hline $2 \mathrm{H}-4,42$ & 14.62 & $\mathrm{AF}$ & 50 & -57.0 & 196.0 & \\
\hline $2 \mathrm{H}-5,18$ & 15.88 & CTD & $510^{\circ}$ & 49.5 & 359.0 & \\
\hline $2 \mathrm{H}-5,44$ & 16.14 & $\mathrm{AF}$ & 80 & 45.0 & 18.0 & \\
\hline $2 \mathrm{H}-6,24$ & 17.44 & AF & 80 & 35.5 & 10.2 & \\
\hline $2 \mathrm{H}-6,50$ & 17.70 & CTD & $515^{\prime \prime}$ & -46.7 & 209.2 & \\
\hline $2 \mathrm{H}-7,7$ & 18.77 & $\mathrm{AF}$ & 45 & -28.3 & 197.0 & \\
\hline $3 \mathrm{H}-1,10$ & 19.30 & $\mathrm{AF}$ & 50 & & & Unstable \\
\hline $3 \mathrm{H}-1,3 \mathrm{I}$ & 19.51 & CTD & $545^{\prime \prime}$ & -29.8 & 28.5 & \\
\hline $3 \mathrm{H}-2,10$ & 20.80 & $\mathrm{AF}$ & 50 & -51.6 & 340.0 & \\
\hline $3 \mathrm{H} \cdot 3,10$ & 22.30 & $\mathrm{AF}$ & 30 & 25.0 & 143.0 & \\
\hline $3 \mathrm{H}-4,10$ & 23.80 & $\mathrm{AF}$ & 80 & -42.0 & 330.0 & \\
\hline $3 \mathrm{H}-4,3 \mathrm{I}$ & 24.01 & CTD & $531^{\circ}$ & -51.9 & 340.0 & \\
\hline $3 \mathrm{H}-5,10$ & 25.30 & $\mathrm{AF}$ & 50 & 44.6 & 141.0 & \\
\hline $3 \mathrm{H}-5,31$ & 25.51 & CTD & $525^{\circ}$ & 56.7 & 150.0 & \\
\hline $3 \mathrm{H}-6,10$ & 26.80 & $\mathrm{AF}$ & 50 & -42.0 & 328.0 & \\
\hline $3 \mathrm{H}-6,3 \mathrm{I}$ & 27.01 & CTD & 544 & -31.8 & 330.0 & \\
\hline $3 \mathrm{H}-7,10$ & 28.30 & $\mathrm{AF}$ & 100 & -30.0 & 317.0 & \\
\hline $4 \mathrm{H}-1,15$ & 28.85 & $\mathrm{AF}$ & 65 & 39.2 & 183.0 & \\
\hline $4 \mathrm{H}-1,58$ & 29.28 & CTD & $532^{*}$ & -7.6 & 265.0 & \\
\hline $5 \mathrm{H}-\mathrm{I}, 30$ & 38.50 & $\mathrm{AF}$ & 50 & & & Unstable \\
\hline $5 \mathrm{H}-1,85$ & 39.05 & CTD & 558 & -8.1 & 235.0 & \\
\hline $5 \mathrm{H}-2,30$ & 40.00 & $\mathrm{AF}$ & 30 & -48.6 & 205.0 & \\
\hline $5 \mathrm{H}-2,85$ & 40.55 & CTD & $559^{\circ}$ & 10.3 & 50.0 & \\
\hline $5 \mathrm{H}-3,85$ & 42.05 & CTD & $547^{\circ}$ & -11.4 & 344.0 & \\
\hline $6 \mathrm{H}-1,40$ & 48.00 & $\mathrm{AF}$ & 50 & -32.0 & 161.0 & \\
\hline $6 \mathrm{H}-2,40$ & 49.50 & $\mathrm{AF}$ & 50 & 28.4 & 185.0 & \\
\hline $6 \mathrm{H}-3,40$ & 51.00 & $\mathrm{AF}$ & 45 & 7.0 & 167.0 & \\
\hline $6 \mathrm{H}-3,91$ & 51.51 & CTD & $511^{\circ}$ & -26.0 & 348.0 & \\
\hline $6 \mathrm{H}-4,40$ & 52.50 & $\mathrm{AF}$ & 40 & & & Unstable \\
\hline
\end{tabular}


Table 3 (continued).

\begin{tabular}{|c|c|c|c|c|c|c|}
\hline Sample $(\mathrm{cm})$ & $\begin{array}{l}\text { Depth } \\
\text { (mbsf) }\end{array}$ & $\begin{array}{l}\text { Demagnetization } \\
\text { type }^{a}\end{array}$ & $\begin{array}{c}\text { Maximum } \\
\text { demagnetization }\end{array}$ & $\begin{array}{c}\text { Inclination } \\
\text { (degrees) }\end{array}$ & $\begin{array}{l}\text { Declination } \\
\text { (degrees) }\end{array}$ & Comments \\
\hline \multicolumn{7}{|l|}{$125-786 \mathrm{~A}-$} \\
\hline $6 \mathrm{H}-4,91$ & 53.01 & CTD & $653^{\circ}$ & 22.1 & 249.0 & \\
\hline $7 \mathrm{H}-1,27$ & 57.37 & $\mathrm{AF}$ & 35 & & & Unstable \\
\hline $7 \mathrm{H}-2,27$ & 58.87 & $\mathrm{AF}$ & 35 & -43.0 & 184.0 & \\
\hline $7 \mathrm{H}-3,27$ & 60.37 & $\mathrm{AF}$ & 25 & & & Unstable \\
\hline $7 \mathrm{H}-4,27$ & 61.87 & $\mathrm{AF}$ & 30 & & & Unstable \\
\hline $7 \mathrm{H}-4,43$ & 62.03 & CTD & $448^{\circ}$ & & & Unstable \\
\hline $7 \mathrm{H}-5,43$ & 63.35 & CTD & $633^{\circ}$ & 26.9 & 138.0 & \\
\hline $7 \mathrm{H}-5,27$ & 63.37 & $\mathrm{AF}$ & 50 & 9.0 & 228.0 & \\
\hline $8 \mathrm{H}-1,19$ & 67.29 & $\mathrm{AF}$ & 50 & -50.0 & 190.0 & \\
\hline $9 \mathrm{H}-1,8$ & 76.88 & AF & 50 & & & Unstable \\
\hline $9 \mathrm{H}-1,114$ & 77.94 & CTD & $475^{\circ}$ & -3.8 & 345.0 & \\
\hline $9 \mathrm{H}-2,73$ & 79.03 & $\mathrm{AF}$ & 50 & -13.0 & 312.0 & \\
\hline $9 \mathrm{H}-3,17$ & 79.97 & $\mathrm{AF}$ & 50 & 4.0 & 239.0 & \\
\hline $9 \mathrm{H}-3,98$ & 80.78 & CTD & $610^{\circ}$ & -8.8 & 4.2 & \\
\hline $9 \mathrm{H}-4,32$ & 81.62 & $\mathrm{AF}$ & 20 & & & Unstable \\
\hline $9 \mathrm{H}-4,114$ & 82.44 & CTD & $641^{\circ}$ & -13.7 & 300.5 & \\
\hline $9 \mathrm{H}-5,9$ & 82.89 & $\mathrm{AF}$ & 30 & & & Unstable \\
\hline $9 \mathrm{H}-5,88$ & 83.68 & CTD & $621^{\circ}$ & -13.9 & 273.0 & \\
\hline $9 \mathrm{H}-6,8$ & 84.38 & $\mathrm{AF}$ & 50 & & & Unstable \\
\hline $9 \mathrm{H}-6,29$ & 84.59 & CTD & $606^{\circ}$ & 43.5 & 317.0 & \\
\hline $10 \mathrm{H}-1,20$ & 86.60 & $\mathrm{AF}$ & 30 & & & Unstable \\
\hline $10 \mathrm{H}-2,23$ & 88.13 & $\mathrm{AF}$ & 20 & & & Unstable \\
\hline $10 \mathrm{H}-2,37$ & 88.27 & CTD & $582^{\circ}$ & -13.7 & 129.0 & \\
\hline $10 \mathrm{H}-3,23$ & 89.63 & $\mathrm{AF}$ & 50 & & & Unstable \\
\hline $10 \mathrm{H}-3,37$ & 89.77 & CTD & $581^{\circ}$ & -30.0 & 322.0 & \\
\hline $10 \mathrm{H}-4,23$ & 91.13 & $\mathrm{AF}$ & 20 & & & Unstable \\
\hline $10 \mathrm{H}-4,37$ & 91.27 & CTD & $504^{\circ}$ & -22.0 & 20.0 & \\
\hline $10 \mathrm{H}-5,18$ & 92.58 & $\mathrm{AF}$ & 40 & & & Unstable \\
\hline $10 \mathrm{H}-6,9$ & 93.99 & $\mathrm{AF}$ & & & & \\
\hline $11 \mathrm{H}-1,19$ & 96.19 & CTD & $522^{\circ}$ & -8.0 & 343.0 & \\
\hline $11 \mathrm{H}-1,22$ & 96.22 & $\mathrm{AF}$ & & & & \\
\hline $11 \mathrm{H}-2,16$ & 97.66 & CTD & $535^{\circ}$ & & & Unstable \\
\hline $11 \mathrm{H}-2,74$ & 98.24 & $\mathrm{AF}$ & & & & \\
\hline $11 \mathrm{H}-3,6$ & 99.06 & CTD & $586^{\circ}$ & & & Unstable \\
\hline $11 \mathrm{H}-3,23$ & 99.23 & $\mathrm{AF}$ & & & & \\
\hline $11 \mathrm{H}-4,11$ & 100.61 & $\mathrm{AF}$ & & & & \\
\hline $11 \mathrm{H}-5,11$ & 102.11 & $\mathrm{AF}$ & & & & \\
\hline
\end{tabular}

Notes: $\mathrm{AF}=$ alternating field $; \mathrm{CTD}=$ continuous thermal demagnetization.

\section{REFERENCES}

Arason, P., and Levi, S., 1990. Compaction and inclination shallowing in deep-sea sediments from the Pacific Ocean. J. Geophys. Res., 95:4501-4510.

Bleil, U., 1982. Paleomagnetism of Deep Sea Drilling Project Leg 60 sediments and igneous rocks from the Mariana region. In Hussong, D. M., Uyeda, S., et al., Init. Repts. DSDP, 60: Washington (U.S. Govt. Printing Office), 855-873.

Celaya, M. A., and Clement, B. M., 1988. Inclination shallowing in deep sea sediments from the north Atlantic. Geophys. Res. Lett., 15:52-55.

Dunn, J. R., and Fuller, M., 1984. Thermal demagnetization with measurements at high temperature using SQUID magnetometer. Eos, 65:863.

Fisher, R. A., 1953. Dispersion on a sphere.Proc. R. Soc. London A, 217:295-305.

Haston, R., and Fuller, M., 1990. Paleomagnetic data from the Philippine Sea plate and their tectonic significance. J. Geophys. Res., 96:6073-6098.

Hirooka, K., Takahasi, T., Sakai, H., and Nakajima, T., 1985. Paleomagnetic evidence of the northward drift of the Izu peninsula, Central Japan. In Nasu, N., et al. (Eds.), Formation of Active Ocean Margins: Tokyo (Terra Sci. Publ.), 775-787.

Keating, B., 1981. Paleomagnetic study of sediments from Deep Sea Drilling Leg 59. In Kroenke, L., Scott, R., et al., Init. Repts DSDP, 59: Washington (U.S. Govt. Printing Office), 523-532.

Keating, B., and Herrero, E., 1981. Paleomagnetic studies of basalts and andesites from Deep Sea Drilling Project Leg 59. In Kroenke, L., Scott,
R., et al., Init. Repts. DSDP, 59: Washington (U.S. Govt. Printing Office), 533-543.

Kinoshita, H., 1980. Paleomagnetism of sediment cores from Deep Sea Drilling Project Leg 58. In Klein, G. deV., Kobayashi, K., et al., Init. Repts. DSDP, 58: Washington (U.S. Govt. Printing Office), 765-768.

Louden, K. E., 1977. Paleomagnetism of DSDP sediments, phase shifting of magnetic anomalies, and rotation of the West Philippine Basin. J. Geophys. Res., 82:2989-3002.

McFadden, P. L., and Reid, A. B., 1982. Analysis of paleomagnetic inclination data. Geophys. J. R. Astron. Soc., 69:307-319.

Niitsuma, N., 1986. Paleomagnetic results, Nankai Trough and Japan Trench. In Kagami, H., Karig, D. E., Coulbourn, W. C., et al., Init. Repts. DSDP, 87: Washington (U.S. Govt. Printing Office), 757-786.

Ranken, B., Cardwell, R. K., and Karig, D. E., 1984. Kinematics of the Philippine Sea plate. Tectonics, 3:555-575.

Zijderveld, J.D.A., 1967. AC demagnetization of rocks: analysis of results. In Collinison, D. W., Creer, K. M., and Runcorn, S. K. (Eds.), Methods in Paleomagnetism: New York (Elsevier), 245-286.

Date of initial receipt: 1 October 1990

Date of acceptance: 19 August 1991

Ms 125B-156 
Table 4. Mean paleomagnetic inclinations from Leg 125.

\begin{tabular}{llcrcrrr}
\hline Hole & \multicolumn{1}{c}{ Age } & $\begin{array}{c}\text { Interval } \\
\text { (mbsf) }\end{array}$ & $N$ & $\begin{array}{c}\text { Inclination } \\
\text { (degrees) }\end{array}$ & \multicolumn{1}{c}{$\alpha_{95}$} & $\kappa$ & $\begin{array}{c}\text { Latitude } \\
\text { (degrees) }\end{array}$ \\
\hline $782 \mathrm{~A}$ & late Pleistocene & $39-54$ & 7 & 45.8 & 7.2 & 22.0 & $27.2 \pm 3.6$ \\
$782 \mathrm{~A}$ & late Miocene & $142-250$ & 20 & 41.7 & 6.0 & 32.0 & $24.0 \pm 3.0$ \\
$782 \mathrm{~A}$ & middle Miocene & $250-280$ & 11 & 40.8 & 7.0 & 52.0 & $23.3 \pm 3.5$ \\
$784 \mathrm{~A}$ & Pliocene & $40-85$ & 73 & 8.5 & 14.0 & 29.0 & $21.7 \pm 7.1$ \\
$786 \mathrm{~A}$ & late Pliocene & $0-15$ & 12 & 51.3 & 9.0 & 36.0 & $32.0 \pm 4.5$ \\
$786 \mathrm{~A}$ & early Pliocene & $15-28$ & 15 & 40.0 & 6.0 & 36.0 & $22.8 \pm 3.0$ \\
\hline
\end{tabular}

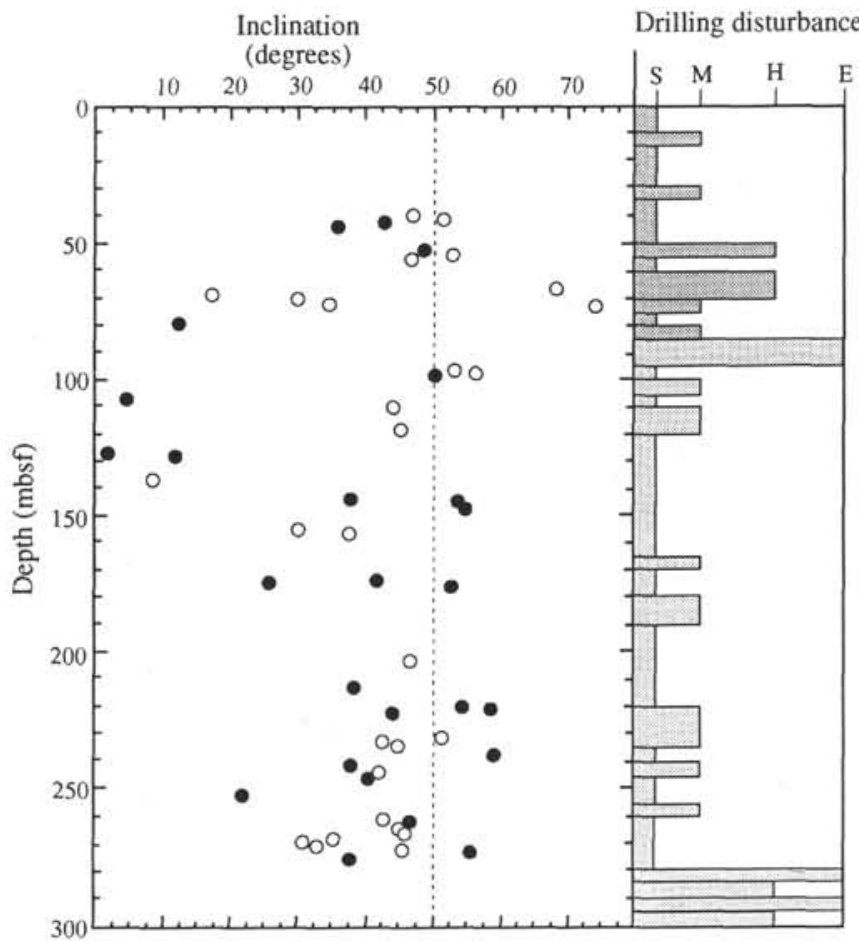

Figure 3. Inclination and drilling disturbance data vs. depth for Hole 782A. Black circles represent normal polarity; open circles indicate the absolute value of the reversed polarity samples. 

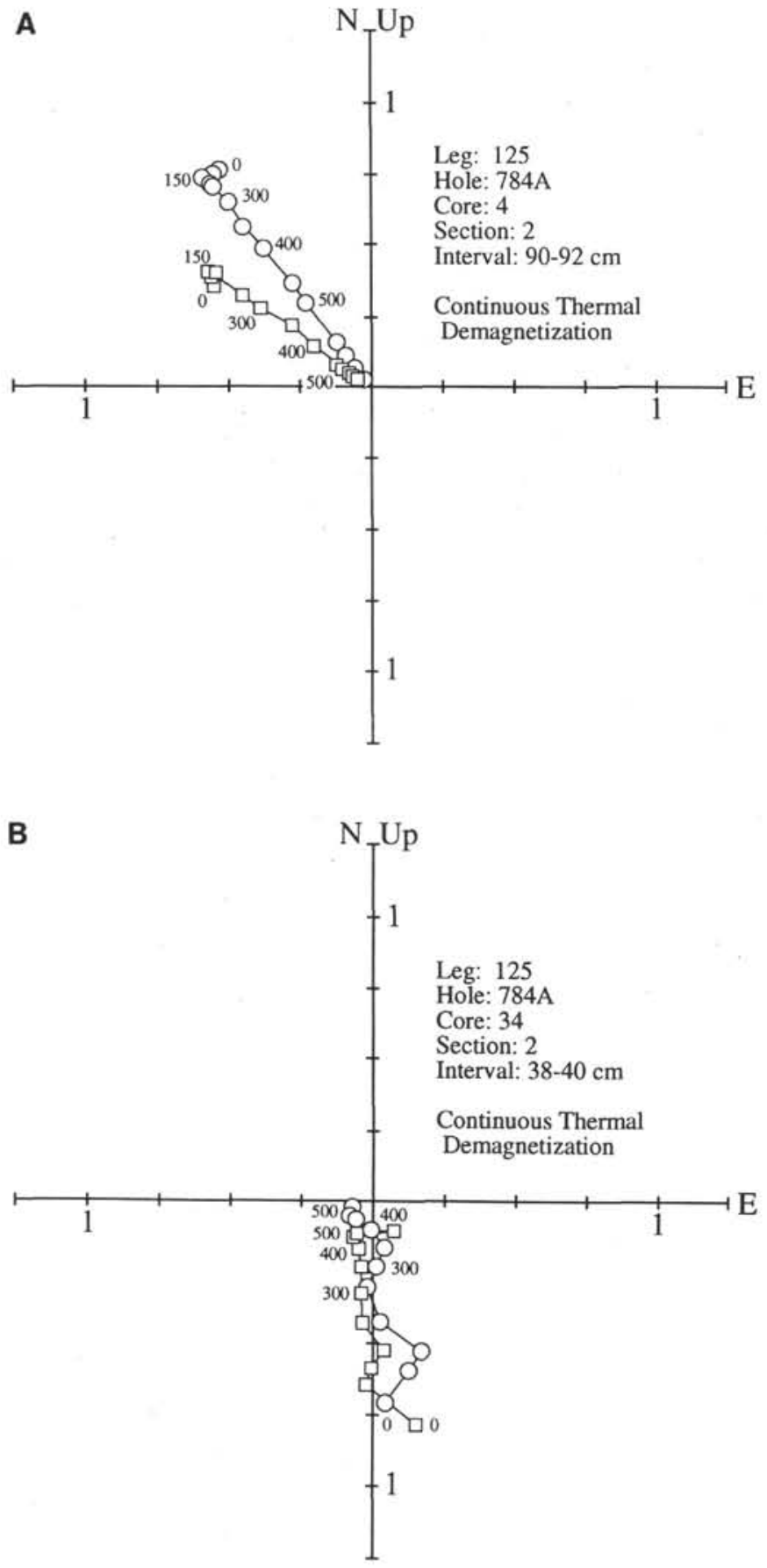

Figure 4. Typical orthogonal vector plots for samples from Hole 784A. A. Single component behavior. B. No characteristic directional behavior.

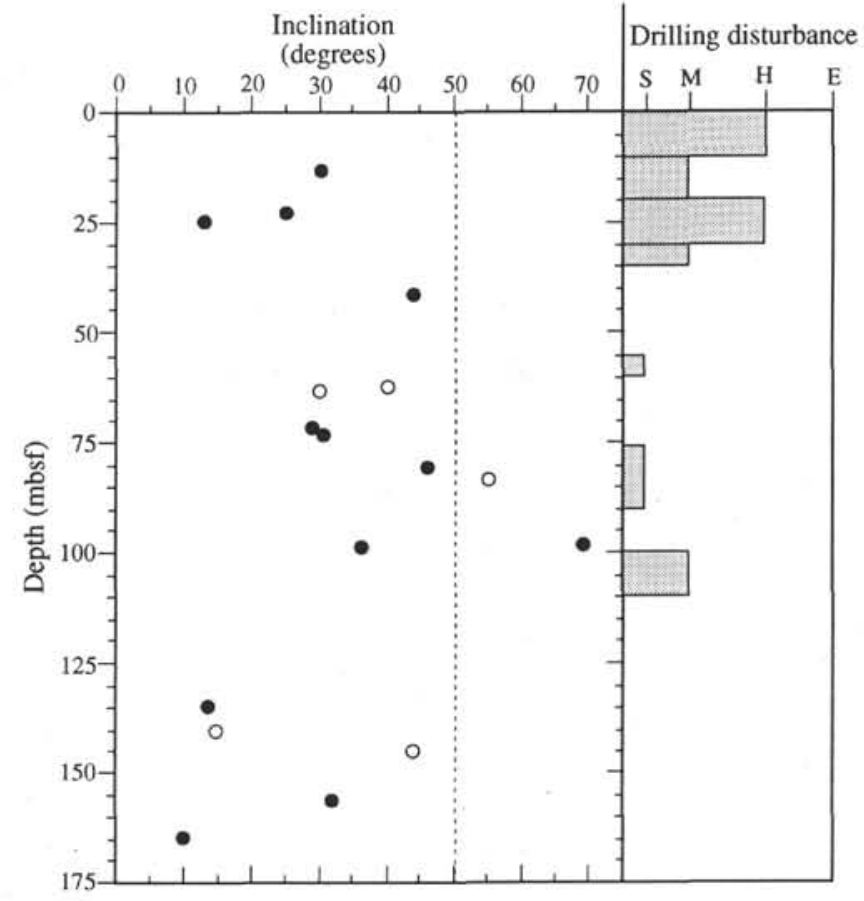

Figure 5. Inclination and drilling disturbance data vs. depth for Hole 784A. Black circles represent normal polarity; open circles indicate the absolute value of the reversed polarity samples. 

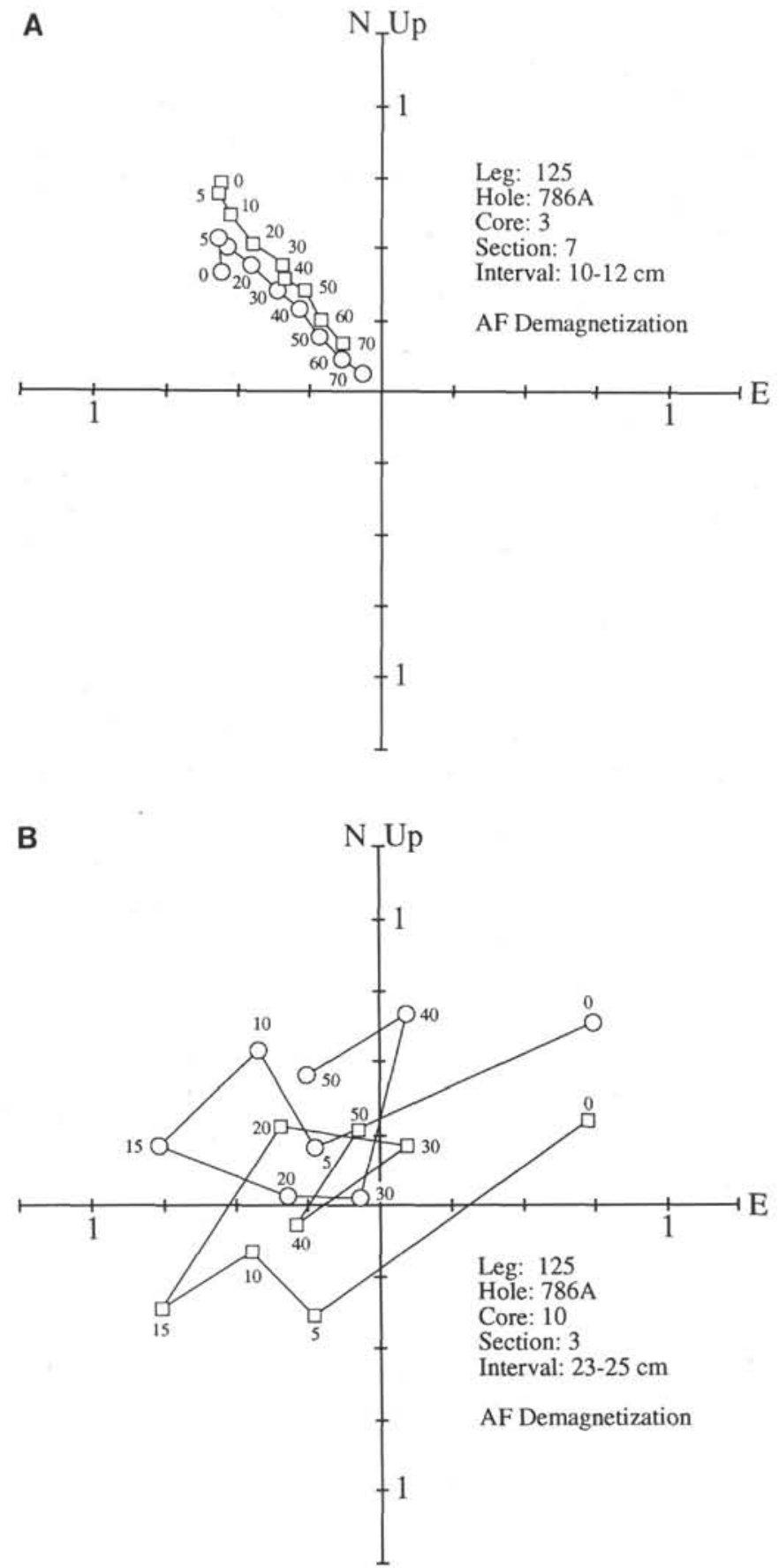

Figure 6. Typical orthogonal vector plots for samples from Hole 786A. A. Single component behavior. B. No characteristic directional behavior.

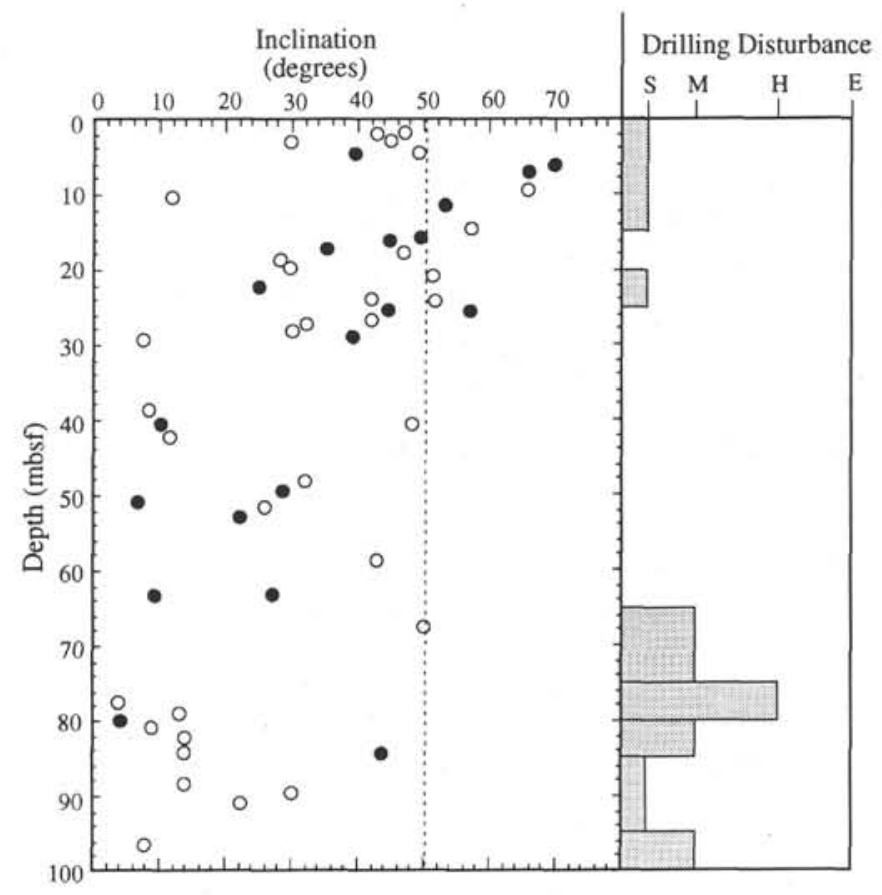

Figure 7. Inclination and drilling disturbance data vs. depth for Hole 786A. Black circles indicate normal polarity; open circles represent the absolute value of the reversed polarity samples. 


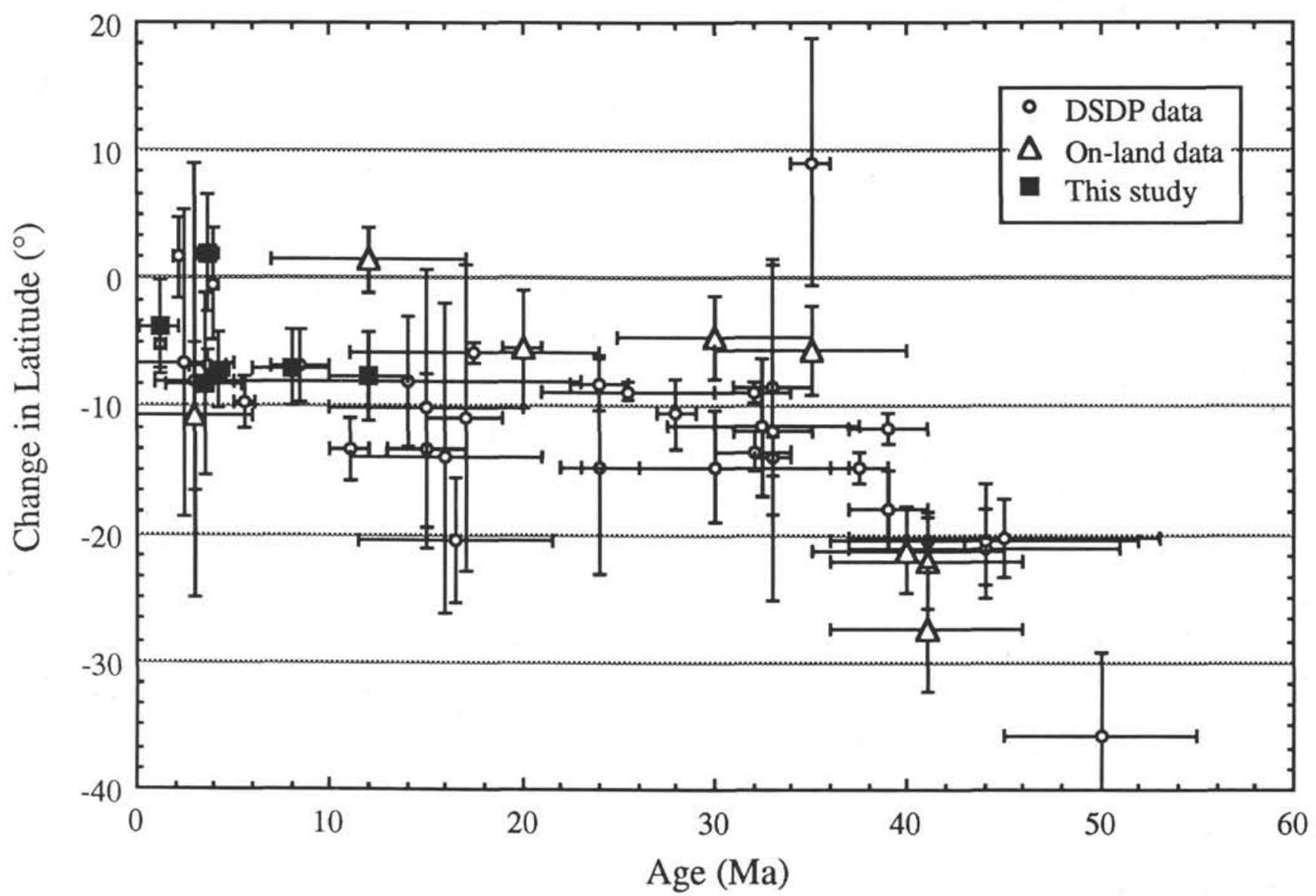

Figure 8. Paleolatitude data from around the Philippine Sea plate. "Change in latitude" is defined as the difference between the observed paleolatitude and the site latitude. 